\title{
JUSTIFICATION OF RATIONAL DESIGN PARAMETERS OF MILKING MACHINE FOR INSTALLATIONS WITH MILK LINE SYSTEM
}

\author{
Oksana Achkevych, Vasyl Achkevych, Viacheslav Bratishko, Svitlana Potapova \\ National University of Life and Environmental Sciences of Ukraine, Ukraine \\ achkevych@gmail.com, achkevychv@gmail.com,vbratishko@gmail.com, \\ svitlapotapova@gmail.com
}

\begin{abstract}
This article is devoted to improving the efficiency of the milking process by improving the mode of milk transport by using a two-chamber milking cluster with partial air intake into the milk collecting chamber in the $d$-phase. Creating a two-chamber cluster with partial air intake will combine the main benefits of operating a milking machine with an alternate and simultaneous pulsator, stabilize the vacuum pressure in the $b$-phase and create a sufficient pressure gradient in the $d$-phase. To establish and verify the design parameters of the twochamber milking cluster, a program and methodology for conducting experimental studies have been developed. Experimental samples of the milking cluster and a laboratory stand with the possibility of varying factors were developed for the research. To determine the influence of factors on the amplitude of the oscillation of the vacuum pressure in the collecting chamber of the cluster in the $b$-phase and in the $d$-phase compression stroke, a screening factor experiment was performed. It is experimentally established that the vacuum pressure resistance in the $b$-phase, within the zootechnical requirements, will be at the diameter of the throttle hole within $2-3 \mathrm{~mm}$, and the diameter of the milk hose more than $12 \mathrm{~mm}$. The use of the milk hose $d_{h}=10 \mathrm{~mm}$ in combination with the small diameter of the throttle hole gives a vacuum variation from 15 to $17 \mathrm{kPa}$. This is characterized by insufficient air supply and does not provide a pressure difference for transporting a portion of milk to the milk line. The use a $12 \mathrm{~mm}$ diameter milk hose creates a vacuum pressure drop from 18 to $11 \mathrm{kPa}$. The use of a $14 \mathrm{~mm}$ diameter milk hose creates a drop in vacuum pressure within the range of 38 to $27 \mathrm{kPa}$, which is not enough to transport the milk-air mixture.
\end{abstract}

Keywords: milking, cluster, vacuum, pressure, milk hose, throttle hole.

\section{Introduction}

High level of development of milking equipment ensures production of quality milk and minimizes the impact on the health of the cow. The question remains of maintaining the quality of milk during transportation from the milking machine to the upper milk line.

The removal of milk from the udder of the cow is caused by the vacuum pressure, the difference in pressure in the udder and the cluster of the milking machine. The transport of milk from the cluster to the milk line requires a pressure gradient, which is created by constant intake of air into the cluster of the milking machine through the throttle hole [1]. This creates a milk-air mixture, and the ratio between the amount of milk and air changes during the milking time. The rational operation of the milking machine is characterized by the stability of the vacuum pressure during withdrawal of milk from the udder with minimal impact on the condition of the milking machine $[2 ; 3]$. According to the zootechnical requirements of milking, the drop in the vacuum pressure during the $b$-phase should not be more than 6-8 $\mathrm{kPa}$, for installations with the upper milk line at the vacuum pressure in the system $46-48 \mathrm{kPa}[4 ; 5]$. The stability of the vacuum pressure in the $b$-phase is influenced by the diameter of the milk hose, the diameter of the throttle hole and the amount of milk entering the collecting chamber of the cluster.

The mismatch between the modes of operation and the design of the milking machine leads to a decrease in the cow productivity, an increase in the incidence of mastitis and a decrease in the quality of milk in general.

The main problem is the instability of the vacuum pressure in the $b$-phase and the insufficient pressure gradient in the $d$-phase. Systematic fluctuations in vacuum pressure have been found to reduce the milk yield by up to $14 \%$, and unstable transport conditions cause milk foaming, dispersion and delamination of fat globules, which are subsequently deposited on the walls of the milk duct and, as a consequence, reduce the milk quality. The fat loss of milk, depending on the length of the milk line, can range from $0.1 \%$ to $0.45 \%[2 ; 4 ; 7]$. 
This problem can be solved by connecting the milking cluster and the pulsator chamber by a throttle hole, which allows to stabilize the vacuum pressure in the $b$-phase and to carry out quality milk transport in the $d$-phase.

Creating a two-chamber milking cluster with partial air inlet will combine the main benefits of operating a milking machine with both an alternative and simultaneous pulsator. Improving the quality of the milk obtained within the zootechnical requirements is possible by stabilizing the vacuum pressure in the $b$-phase and creating a sufficient pressure gradient in the $d$-phase.

A characteristic design feature of the milking cluster in Fig. 1 is the presence of two milking chambers, each of which connects to the milk line through a separate milk hose and collects milk from only two milking cups. In this case, each milk collection chamber is connected by a throttle hole to the respective distributor chambers of the collector. This solution will improve the mode of transportation of milk, stabilize the pressure in the milk cluster during the $b$-phase, and ensure its effective "purging" during the $d$-phase.
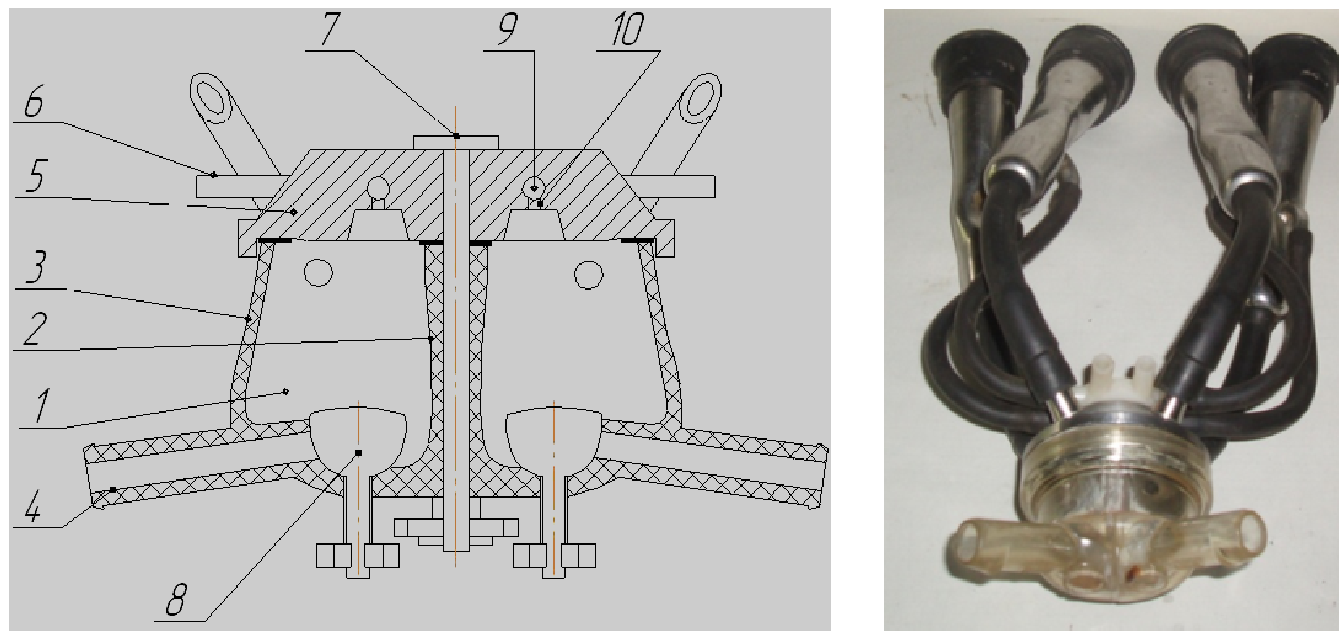

Fig. 1. Scheme of the developed milking cluster and general appearance: 1 - milk chamber;

2 - partition; 3 - case; 4 - cluster outlet pipe; 5 - cover with the air distribution chamber; 6 - air nozzles to the interstitial spaces of milking glasses; 7 - clamping bolt; 8 - shut-off valve; 9 - air pipe to the pulsator; 10 - throttle hole

\section{Materials and methods}

Experimental simulation of milking and milk transport was used for experimental studies. The general view is shown in Fig. 2, and the scheme of the test stand in Fig. 3.

The features of this design when conducting experiments are:

- portion air supply to the milking cluster through the throttle hole,

- milk removal is carried out on two independent hoses,

- operation of such advanced milking machine combines alternative and simultaneous operation of the milking machine.

A factor experiment was conducted to determine the effect of the elements (diameter of the milk hose $d_{h}$, the diameter of the throttle hole $d_{t}$, the milk flow rate $Q_{m}$ ) on the level of the vacuum pressure in the milking cluster chamber in the $b$-phase and in the $d$-phase.

The studies were performed according to the provisions of ISO 6690: 2004 and JMA 74.3-37-273: 2005 with a triple repetition of experiments.

In the course of the research milk hoses were used with the inner diameters of 10,12 and $14 \mathrm{~mm}$. The intensity of the milk yield varied from $0.21 \cdot \mathrm{min}^{-1}$ to $31 \cdot \mathrm{min}^{-1}$ by means of a liquid feed valve and monitored by a rotameter. To change the throttle hole in the manifold lid, inserts with holes 1, 2 and $3 \mathrm{~mm}$ were used. The working vacuum pressure in the system was $48-50 \mathrm{kPa}$. 


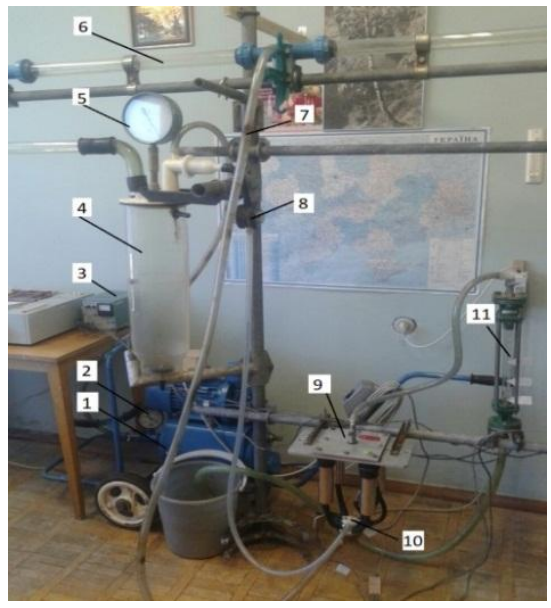

Fig. 2. General view of the installation for experimental studies: 1 - vacuum installation; $2 ; 5$ - vacuum meter; 3 - self-recording device; 4 - water tank (milk collector); 6 - milk line; 7 - milk hose; 8 - pulsator; 9 - artificial udder;

10 - suspension part of the milking machine; 11 - rotameter

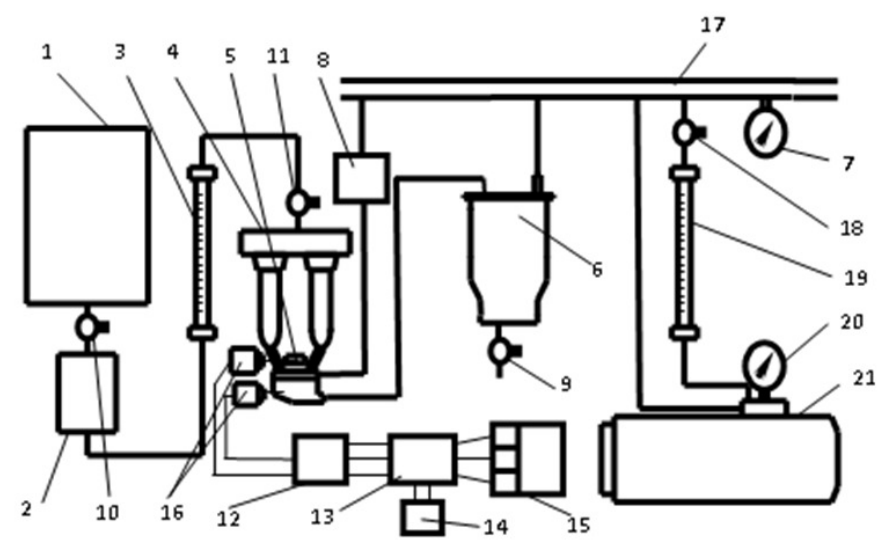

Fig. 3. Scheme of the test stand for the study of the modes of operation of the milking machine: $1-\operatorname{tank}$; 2 - milk simulator; 3 - rotameter; 4 - artificial udder; 5 - suspended part of the milking machine; 6 - water tank (milk collector); 7.20 - vacuum meter; 8 - pulsator; 9 , 10, 11, 18 - crane; 12 - comparison unit; 13 - amplifier; 14 - power supply; 15 - self-recording device;

16 - tensometric pressure sensors; 17 - vacuum conduit; 19 - air flow meter; 21 - receiver

\section{Results and discussion}

As the result of the experimental studies, equations of the change of the vacuum pressure were obtained depending on the change of the design parameters of the milking machine.

As shown by the experimental studies, the amplitude of the vacuum pressure in the milking cluster chamber in the $b$-phase is described by the dependence

$$
P_{k c c}=7.083+27.068 d_{t}-2.696 d_{t}^{2}-1.252 d_{h} d_{t}-1425.737 Q_{m}{ }^{2}+0.175 d_{h}{ }^{2},
$$

Where $d_{t}$ - diameter of the throttle hole, $\mathrm{m}$;

$d_{h}$ - diameter of the milk hose, m;

$Q_{m}-$ milk flow rate, $\mathrm{m}^{3} \cdot \mathrm{s}^{-1}$.

For dependence (1), which is adequate at $95 \%$ confidence level, the multiple determination factor $D=0.872301$, the multiple correlation coefficient $R=0.934247$. All given model coefficients are significant at a confidence level of at least $95 \%$.

Analysis of the model (1) indicates the nonlinear nature of the dependence of the vacuum pressure $P_{k c c}$ on the diameter of the milk hose $d_{h}$ and the diameter of the throttle hole $d_{t}$. Increasing the diameter of the milk hose provides better stabilization of the vacuum pressure. This indicates that there is sufficient residual air in the milking cluster chamber due to the larger volume of the milk line.

Analysis of the graphical dependencies (Fig. 4) shows that the stability of the vacuum pressure in the b-phase within the zootechnical requirements $(40 \mathrm{kPa})$ is observed, when using the diameter of the throttle hole more than $d_{t}=2 \mathrm{~mm}$, regardless of the diameter of the milk hose. The diameter of the throttle hole $d_{t}=1 \mathrm{~mm}$ is possible with the use of a milk hose with a diameter greater than $d_{h}=12.5 \mathrm{~mm}$. The use of a milk hose $d_{h}=10 \mathrm{~mm}$ in combination with a small diameter throttle hole gives oscillations $P_{k c c}$ up to $34 \mathrm{kPa}$. This indicates that there is insufficient volume of air in the milking device.

Analysis of the graphical dependence (Fig. 5) confirms the rationality of the choice of the throttle hole within $d_{t}=2-2.5 \mathrm{~mm}$, which provides maximum stability of the vacuum pressure in combination with the diameter of the milk hose $d_{h}=12 \mathrm{~mm}$. The drop in the vacuum pressure at $d_{h}=14 \mathrm{~mm}$ is up to $P_{k c c}=46 \mathrm{kPa}$, for $d_{h}=12 \mathrm{~mm}$-up to $P_{k c c}=42 \mathrm{kPa}$, and for $d_{h}=10 \mathrm{~mm}$-up to $P_{k c c}=40 \mathrm{kPa}$, which meets the zootechnical requirements. 


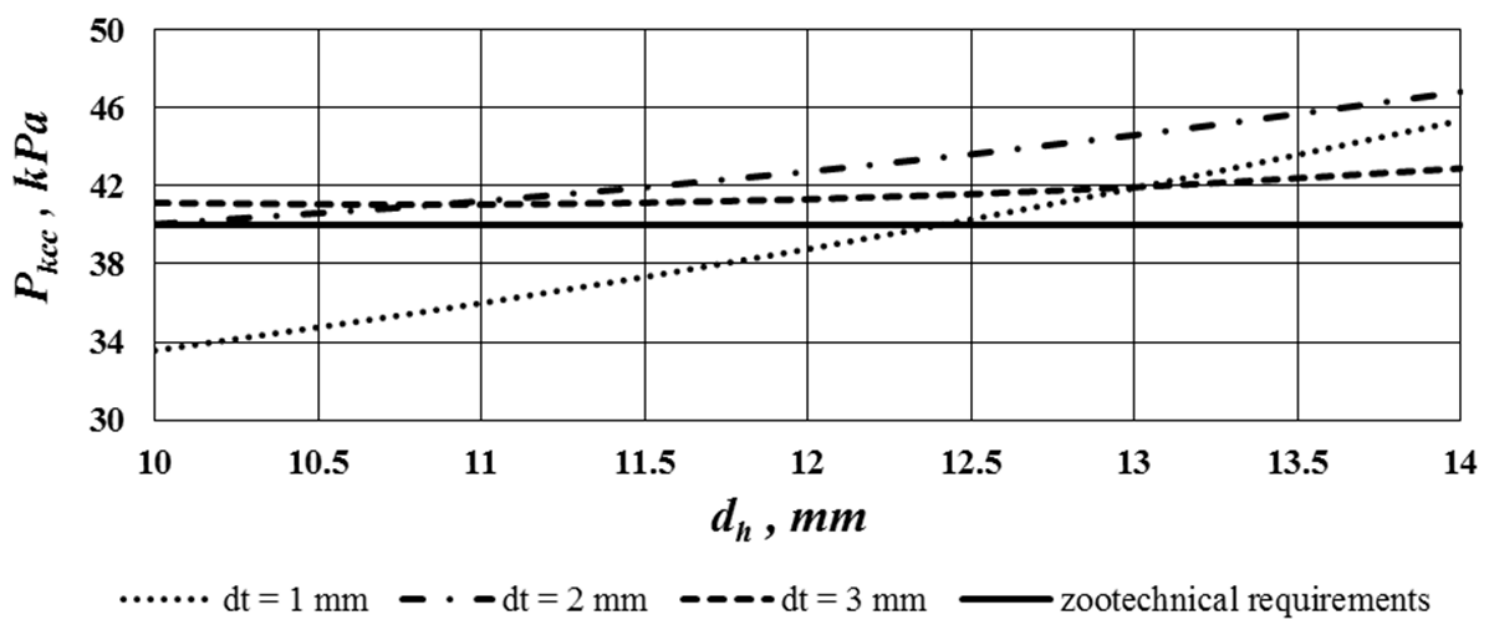

Fig. 4. Changing the vacuum pressure in the milking cluster chamber in the $b$-phase from the diameter of the $d_{h}$ of the milk tube to the milk flow rate $Q_{m}=31 \cdot \mathrm{min}^{-1}$

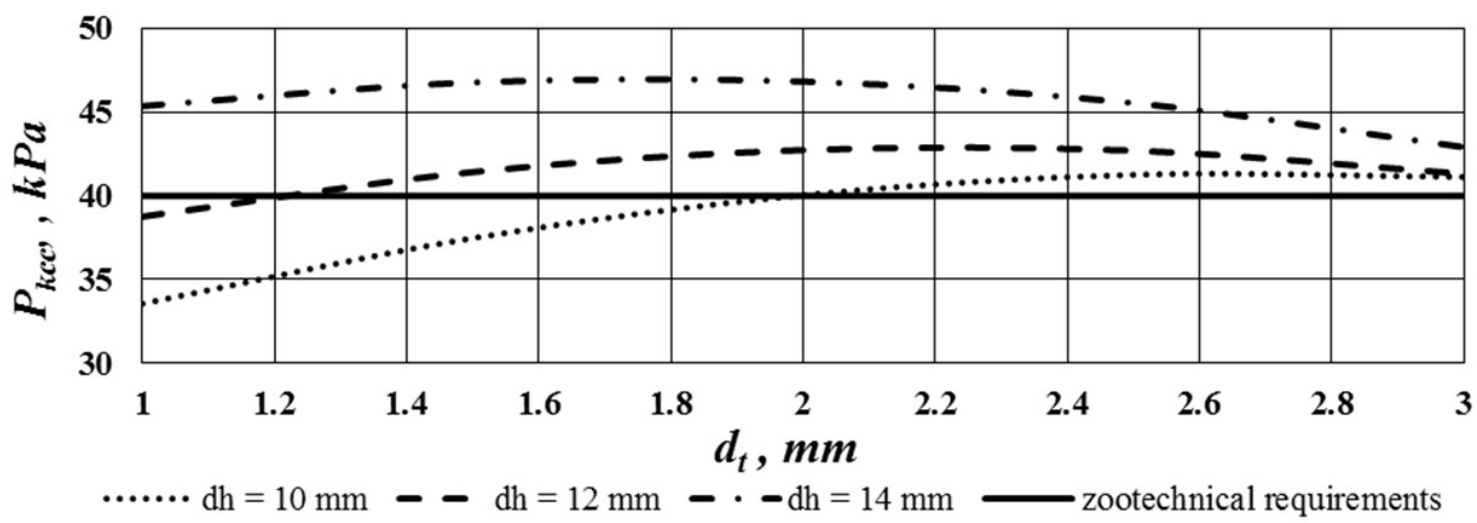

Fig. 5. Changing the vacuum pressure in the milking cluster chamber in the $b$-phase from the diameter of $d_{t}$ the throttle hole with the milk flow rate $Q_{m}=31 \cdot \mathrm{min}^{-1}$

The $d$-phase is responsible for the process of transporting a portion of milk from the chamber of the milking cluster to the milk line. This is due to the pressure difference that creates the air that has come in through the throttle hole. A regression equation was obtained describing the change in the vacuum pressure in the chamber of the milking cluster in the $d$-phase $P_{k s t}$ depending on the factors (2):

$$
P_{k s t}=142.188+8.410 d_{t}-27.072 d_{h}-0.987 d_{h} d_{t}-21.262 Q_{m} d_{h}+1.507 d_{h}^{2},
$$

For the dependence (2), which is adequate at $95 \%$ confidence level, the coefficient of multiple determination $D=0.948352$, the coefficient of multiple correlation $R=0.974211$. All given model coefficients are significant at a confidence level of at least $97 \%$.

From the analysis of the graphical dependencies (Fig. 6), the minimum value of the vacuum pressure $P_{k s t}=15 \mathrm{kPa}$ (zootechnical requirements) is reached with the diameter of a milk hose $d_{h}=12 \mathrm{~mm}$ and a throttle hole with the diameter $d_{t}=2 \mathrm{~mm}$. The diameter of the $d_{h} \leq 12 \mathrm{~mm}$ increases the probability of milking machine falling from the udder, and the diameter of the $d_{h} \geq 12 \mathrm{~mm}$ causes a deterioration in the quality of milk transport.

The graphical dependence of the change of the vacuum pressure $P_{k s t}$ on the diameter of the throttle hole is shown in Fig. 7. The analysis confirms that the use of $d_{h} \leq 12 \mathrm{~mm}$ creates a drop in the vacuum pressure $P_{k s t}$ up to $7-10 \mathrm{kPa}$, regardless of the diameter of the throttle opening. The application of $d_{h}=14 \mathrm{~mm}$ creates a drop in the vacuum pressure $P_{k s t}$ up to $30 \mathrm{kPa}$ for $d_{t}=1 \mathrm{~mm}$, and up to $27 \mathrm{kPa}$ for $d_{t}=3 \mathrm{~mm}$.

Vacuum pressure drops are of greater importance as the diameter of the milk-hose is reduced. The critical level of vacuum pressure is at the diameter of the milk-hose $d_{h}=10 \mathrm{~mm}$, regardless of the diameter of the throttle hole. 


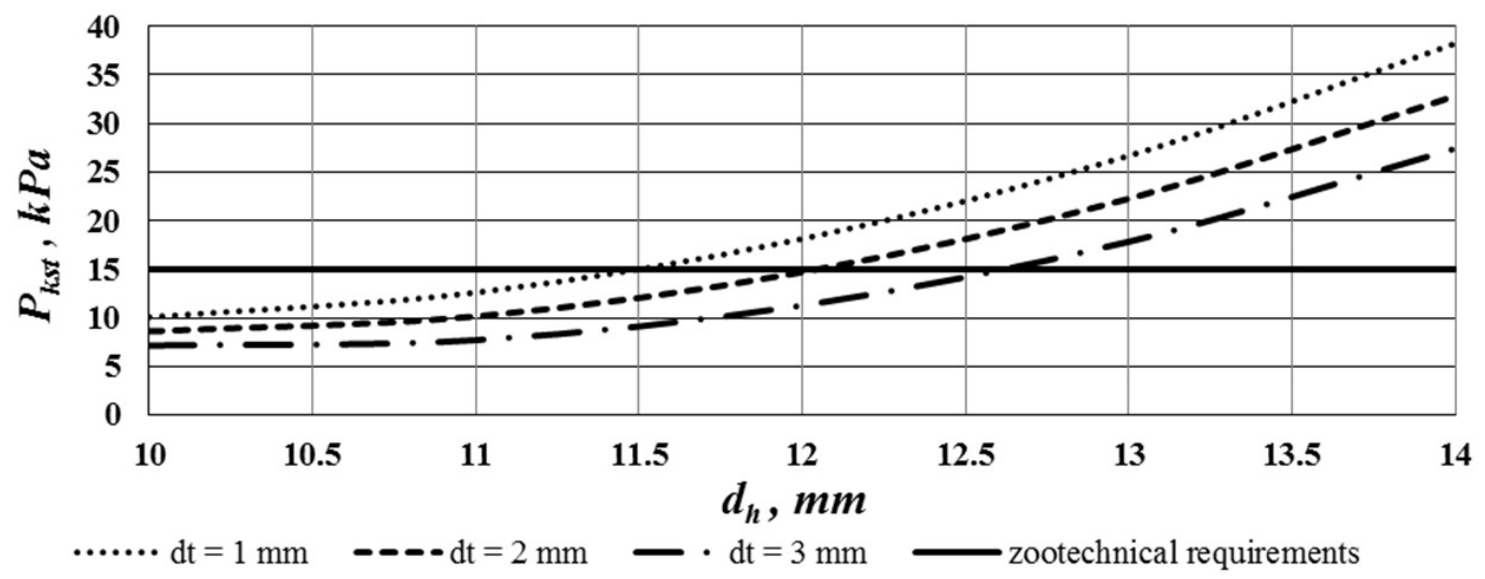

Fig. 6. Dependence of the vacuum pressure in the chamber of the milking cluster in the $d$-phase on the diameter of the milk hose $d_{h}$ at the milk flow rate $Q_{m}=31 \cdot \mathrm{min}^{-1}$

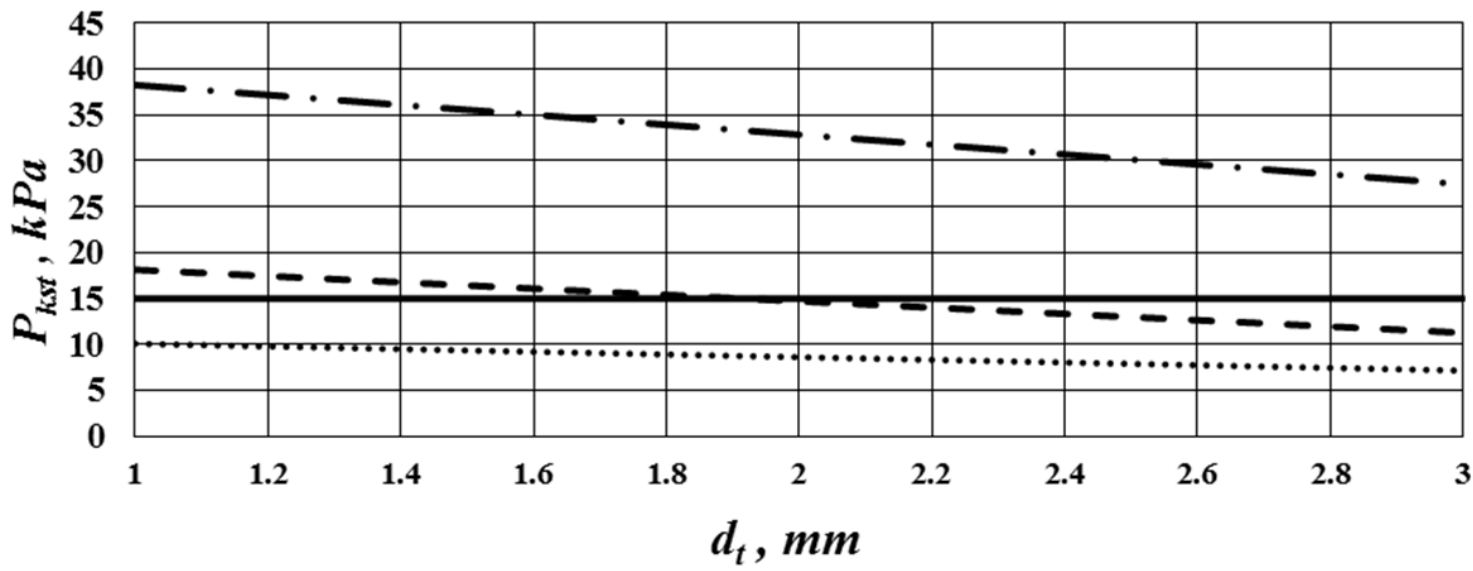

…. dh $=10 \mathrm{~mm} \quad-\quad \mathrm{dh}=12 \mathrm{~mm} \quad-\quad \mathrm{dh}=14 \mathrm{~mm} \quad$ zootechnical requirements

Fig. 7. Dependence of the vacuum pressure in the chamber of the milking cluster in the $d$-phase on the diameter of the throttle hole $d_{t}$ at the milk flow rate $Q_{m}=31 \cdot \mathbf{m i n}^{-1}$

The analysis of the results of the study shows that sufficient fluctuations in the vacuum pressure and transport of the milk-air mixture will be used, when using a milk hose with a diameter $d_{h}=12 \mathrm{~mm}$ in combination with the diameter of the throttle hole within 1.8-2.2 $\mathrm{mm}$. This will result in a drop in the vacuum pressure within the range of $18-15 \mathrm{kPa}$. When using a milk hose with a diameter $d_{h}=14 \mathrm{~mm}$, the drop in vacuum pressure will be within the range of $38-27 \mathrm{kPa}$, which is not sufficient for reliable transportation of the milk-air mixture. When using a milk hose with a diameter of $d_{h}=10 \mathrm{~mm}$, the drop in vacuum pressure will have critical limits of $10-7 \mathrm{kPa}$ (absolute pressure value of $90-93 \mathrm{kPa})$.

\section{Conclusions}

1. It has been experimentally established that the stability of the vacuum pressure in the $b$-phase within the zootechnical requirements $(40 \mathrm{kPa})$ will be in the diameter of the throttle hole $2-3 \mathrm{~mm}$, regardless of the diameter of the milk hose. Using the diameter of the throttle hole $1 \mathrm{~mm}$ is possible, when using the diameter of the milk hose greater than $12.5 \mathrm{~mm}$.

2. According to the analysis of the vacuum pressure in the $d$-phase, it was found that the optimum pressure value is achieved with a milk hose diameter of $12 \mathrm{~mm}$ and a throttle hole diameter within $2 \mathrm{~mm}$. Increasing the diameter of the milk hose over $14 \mathrm{~mm}$, creates a pressure drop of up to $30 \mathrm{kPa}$, which is not enough to transport a portion of milk. Reducing the diameter of the milk hose to less than $12 \mathrm{~mm}$, will cause an excess pressure drop of up to $10 \mathrm{kPa}$.

3. The rational design parameters of the milking cluster, with a portion air inlet, are the diameter of the milk hose $12 \mathrm{~mm}$ and the diameter of the throttle hole $2 \mathrm{~mm}$. 


\section{References}

[1] Rasmussen M. D., Wiking L., Bjerring M., Larsent H. Influence of Air Intake on the Concentration of Free Fatty Acids and Vacuum Fluctuations During Automatic Milking. Journal of Dairy Science, vol. 89, 2006, pp. 4596-4605.

[2] Besier J., Lind O., Bruckmaier R. Dynamics of teat-end vacuum during machine milking: types, causes and impacts on teat condition and udder health. Journal of Applied Animal Research, vol. 44, 2016, pp. 263-272.

[3] Ambord S., Bruckmaier R. Milk flow-dependent vacuum loss in high-line milking systems: Effects on milking characteristics and teat tissue condition. Journal of Dairy Science, vol. 93, issue 8, 2010, pp. 3588-3594.

[4] Hanus O., Vegricht J., Frelich J., Macek A. Analysis of raw cow milk quality according to free fatty acid contents in the Czech Republic. Czech Jornal Animal Science, vol. 53, 2008, pp. 17-26.

[5] Jastova B. Quality of raw milk from a farm with automatic milking system in the Czech Republic. Acta Veterinaria Brno, vol. 80, 2011, pp. 207-214.

[6] Tremblay M., Hess J., Christenson M. Factors associated with increased milk production for automatic milking systems. Journal of Dairy Science, vol. 99, issue 5. 2016, pp. 3824-3837.

[7] Steven C., Nicole H. Influence of raw milk quality on processed dairy products: How do raw milk quality test results relate to product quality and yield? Journal of Dairy Science, vol. 99, issue 12, 2016, pp. 10128-10149. 